\title{
Important research outcomes for treatment studies of perinatal depression: systematic overview and development of a core outcome set
}

Christel Hellberg ${ }^{1}$, Marie Österberg ${ }^{1}$, AnnKristine Jonsson $^{1}$, Sara Fundell ${ }^{1}$, Frida

Trönnberg ${ }^{2}$, Maria Jonsson ${ }^{3}$, and Alkistis Skalkidou ${ }^{4}$

${ }^{1}$ Swedish Agency for Health Technology Assessment and Assessment of Social Services, Stockholm, Sweden

${ }^{2}$ Patient representative

${ }^{3}$ Department of Women's and Children's Health, Uppsala University

${ }^{4}$ Affiliation not available

December 15, 2020

\begin{abstract}
Objective To develop a Core Outcome Set (COS) for treatment of perinatal depression Design Systematic overview of outcomes reported in the literature and consensus development study using a Delphi survey and modified nominal group technique. Setting International. Population Two hundred and twenty-two participants, representing thirteen countries. Methods A systematic overview of outcomes reported in recently published research, a two-round Delphi survey, a consensus meeting at which the final COS was decided. Main results In the literature search, 1772 abstracts were identified and evaluated, 284 papers/protocols were assessed in full and 165 studies were finally included in the review. In all, 106 outcomes were identified and thus included in the Delphi survey. 222 participants registered for the first round of the Delphi survey and 151 (68\%) responded. In the second round, 123 (55\%) participants responded. The following 9 outcomes were agreed upon for inclusion in the final COS: self-assessed symptoms of depression, diagnosis of depression by a clinician, parent to infant bonding, self-assessed symptoms of anxiety, quality of life, satisfaction with intervention, suicidal thoughts, attempted or committed suicide, thoughts of harming the baby, and adverse events. Conclusions The relevant stakeholders prioritised outcomes and reached consensus on a COS comprising nine outcomes. We hope that this COS will contribute to consistency and uniformity of outcome selection and reporting in future clinical trials involving treatment of perinatal depression Funding This article is adapted from a report by SBU, which provided funding for the study. Keywords: perinatal depression, postpartum depression, antenatal depression, COS
\end{abstract}

\section{Hosted file}

Treatment of perinatal depression final.pdf available at https://authorea.com/users/ 382942/articles/498818-important-research-outcomes-for-treatment-studies-of-perinataldepression-systematic-overview-and-development-of-a-core-outcome-set 


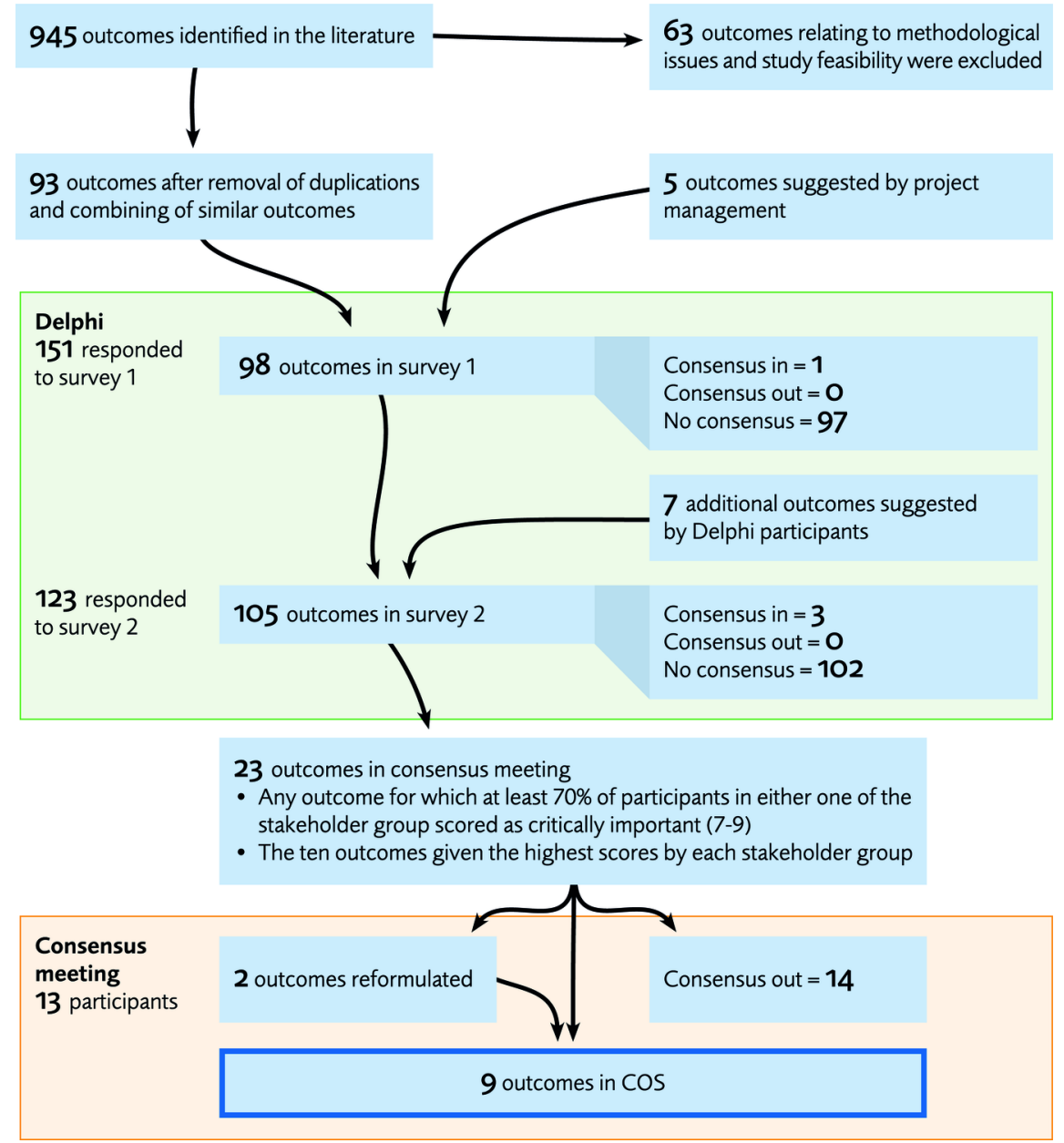

\section{Hosted file}

Table 1.pdf available at https://authorea.com/users/382942/articles/498818-importantresearch-outcomes-for-treatment-studies-of-perinatal-depression-systematic-overviewand-development-of-a-core-outcome-set

\section{Hosted file}

Table 2.pdf available at https://authorea.com/users/382942/articles/498818-importantresearch-outcomes-for-treatment-studies-of-perinatal-depression-systematic-overviewand-development-of-a-core-outcome-set 


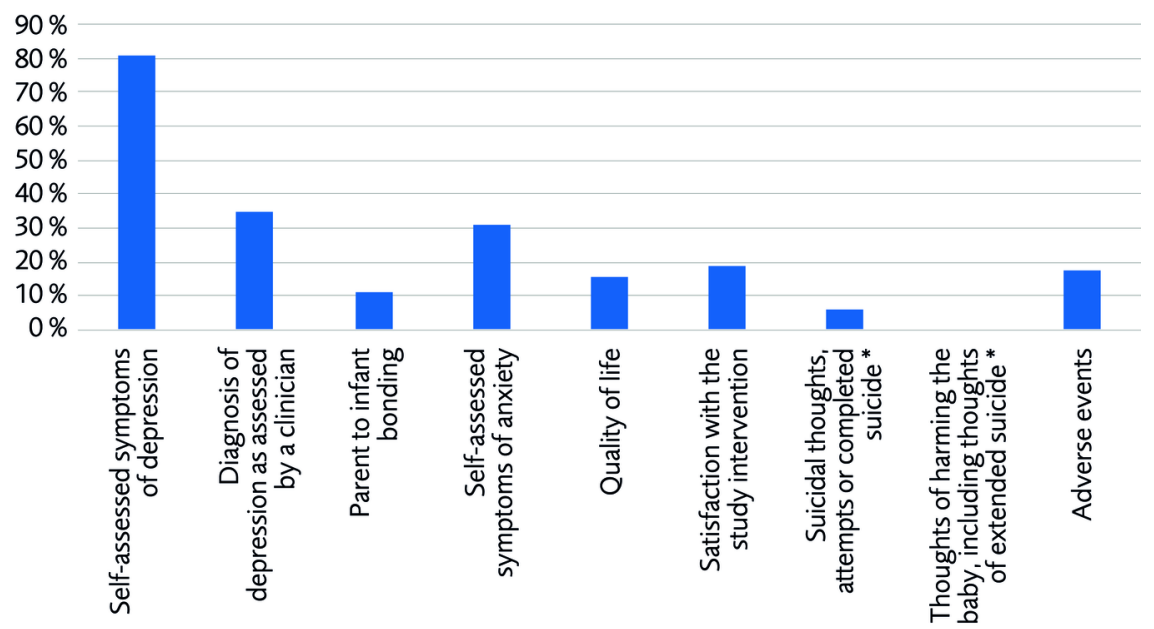

